\title{
PV system sizing using observed time series of solar radiation
}

\author{
T. Markvart ${ }^{\mathrm{a}, *}$, A. Fragaki ${ }^{\text {a }}$, J.N. Ross ${ }^{\mathrm{b}}$ \\ ${ }^{a}$ School of Engineering Sciences, University of Southampton, Southampton SO17 1BJ, UK \\ ${ }^{\mathrm{b}}$ School of Electronics and Computer Science, University of Southampton, Southampton SO17 1BJ, UK
}

Received 20 January 2005; received in revised form 1 August 2005; accepted 8 August 2005

Available online 10 October 2005

Communicated by: Associate Editor Aaron Sanchez-Juarez

\begin{abstract}
Sizing represents an important part of photovoltaic system design. This paper describes a sizing procedure based on the observed time series of solar radiation. Using a simple geometrical construction, the sizing curve is determined as a superposition of contributions from individual climatic cycles of low daily solar radiation. Unlike the traditional methods based on loss-of-load probability, the reliability of supply enters in this method through the length of the time series of data used in the analysis. The method thus resembles techniques used in other branches of engineering where extreme values are considered as functions of certain recurrence intervals.
\end{abstract}

(C) 2005 Elsevier Ltd. All rights reserved.

Keywords: Photovoltaic systems; Sizing; Solar radiation

\section{Introduction}

The sizing of stand-alone photovoltaic (PV) systems is an important part of the system design, and remains an active area for research (see, for example, a review of the sizing tools in Silvestre (2003). In the most fundamental form, sizing procedures consider the relationship between the sizes of the PV array and the battery which delivers energy to load with a certain reliability of supply which can be tolerated by the user. The result of the sizing

\footnotetext{
${ }^{*}$ Corresponding author. Tel.: +442380 593783; fax: +442380 593016.

E-mail address: tm3@soton.ac.uk (T. Markvart).
}

procedure can often be summarised in the form of a sizing curve.

A satisfactory definition of supply reliability is key to this analysis. Loss of load probability (LLP) - the traditional power engineering indicator-is most often used for this purpose, but other descriptions have also been given (Silvestre, 2003). The subject was reviewed in a particularly clear form by Gordon (1987). A more recent review was given by Egido and Lorenzo (1992) (see also Lorenzo et al., 1994, Chapter 6) who used an elaboration of the methods of Barra et al. (1984) and Bartoli et al. (1984) to simulate the operation of a PV systems in a number of Spanish sites, and tabulated the results for the sizing curve, including the dependence on the loss-of-load probability as a 
parameter. Bucciarelli $(1984,1986)$ developed an elegant sizing method based on the principles of random walk. Markvart (1996) used the sizing curve to investigate the sizing of $\mathrm{PV} /$ wind hybrid systems.

The construction of a sizing curve based on LLP requires the modelling of $\mathrm{PV}$ system operation over substantial periods of time. Time series of solar radiation then cannot come directly from observation but need to be reproduced "synthetically" based on an algorithm which is faithful to the solar radiation statistics. The relationship between the LLP values and the perceived reliability requirements of the user are then indirect, although generally accepted correspondence exist for most standard applications (Lorenzo et al., 1994).

This paper develops a somewhat different approach to the reliability of supply, and the use of this concept to size a PV system. The method is based on a direct use of solar radiation data near the site where the PV system is to be installed. By using a standard model based on daily energy balance (see, for example, Fragaki, 2005; Fragaki and Markvart, 2005) configurations can be determined, defining a system that will provide power without shedding load over the period of time when the data are available.

For sites with a good availability of daily solar radiation data, this approach offers several advantages over the traditional LLP-based methods. In the first instance, it obviates the need to produce synthetic time series of solar radiation data. The concept of reliability is also brought closer to the user's experience, by being linked to a duration of time over which the system is expected to operate with $100 \%$ reliability. Clearly, a system designed to operate over 50 years will have a higher reliability (by virtue of a larger PV array and/or a larger battery bank) than a system designed using 10 years of data.

In this approach, the method follows the established engineering practice (used, for example, to asses the effect of extreme winds on building structures or in the design of flood protection measures (Castillo, 1988) where extreme values are considered as functions of certain recurrence intervals. The reader may be familiar with notions such as 50 year wind or 100 year flood, although similar periods for PV systems in specific applications have not been established with any degree of certainty.

The methodology proposed in this paper is based on the analysis of PV system operation as a function of time, portrayed as a series of climatic cycles
(Lorenzo et al., 1994). The relevant climatic cycles are considered as independent, as confirmed by a more detailed analysis (Fragaki, 2005). Thus, only the most significant cycles need be brought into the analysis, and the result taken as their linear superposition. Which cycles are "significant" is determined by modelling, as already mentioned above. With some experience, however, an inspection of the time series may well be sufficient to pinpoint the likely periods which can then be subjected to a closer scrutiny based on a relatively simple mathematical analysis.

The method is illustrated by sizing a PV system near London, UK, using 10 years of measured daily solar radiation data between 1980 and 1990 obtained from the European Solar Radiation Atlas (Scharmer and Grief, 2000).

\section{Sizing curve construction}

To describe the operation of a stand-alone PV system, we follow the usual practice and consider the long-term energy balance between the energy generated by the PV array, the energy consumed by the load, and the energy stored in a battery. We shall denote by $G_{\mathrm{d}}$ the daily solar radiation incident on the plane of the array, and by $L$ the energy consumed by the load in one day. The size of the PV array $P_{0}$ is equal to the product of the array area and its conversion efficiency tested under standard irradiance of $1 \mathrm{~kW} / \mathrm{m}^{2}$. The units of $P_{0}$ are then conventionally given as $W_{\mathrm{p}}$ and the daily solar radiation used to size the system is expressed in Peak Solar Hours, with the implied dimension of time (Castañer et al., 2003). For simplicity, the daily load $L$ is assumed to be constant, and that all the power is consumed at night.

The PV system design would be a simple matter if there were no day-to-day variation of solar radiation. With $G_{\mathrm{d}}$ constant (and equal to $G_{\mathrm{d} 0}$, say), the energy required to power the load would be supplied by an array of size

$P_{0}=\frac{L}{G_{\mathrm{d} 0}}$

In this instance, a rechargeable battery would only be needed to store sufficient energy to cover the daily mismatch between the energy consumption and supply.

It is the variable nature of solar radiation that introduces an uncertainty into how much energy can be provided on a given day. This, in turn, brings 
in the necessity to equip the PV system with a battery (or another type of energy storage) to cope with any significant period of low solar radiation. Let us consider, for example, a time interval of $n_{\mathrm{c}}$ days when the daily solar radiation is equal to $G_{\mathrm{d}}$, below the average value of $G_{\mathrm{d} 0}$. During this time interval (or climatic cycle), a part or all of the load demand has to be supplied by the battery. To maintain a continuous electricity supply to the load, the required battery size $B$, in energy units, must satisfy

$B \geqslant n_{\mathrm{c}}\left(L-P_{0} G_{\mathrm{d}}\right)$

We should note that the battery size $B$ contains only the climatic component of energy storage. The full storage capacity is obtained by adding the regular daily mismatch, in this case, the full load $L$ since all the load occurs at night.

It is usual (Egido and Lorenzo, 1992) to express the array size as a dimensionless multiple $C_{\mathrm{A}}$ of the array size (1) required to supply the load during the average irradiation:

$P_{0}=C_{\mathrm{A}} \frac{L}{G_{\mathrm{d} 0}}$

In some works (see, for example, Klein and Beckman, 1987) the quantity $C_{\mathrm{A}}$ is called the solar-toload ratio (SLR).

If the battery size $B$ is replaced by the days of storage $C_{\mathrm{S}}=B / L$, the condition given in the inequality (2) for continuity of supply can be written as

$\frac{1}{n_{\mathrm{c}}} C_{\mathrm{S}}+\frac{G_{\mathrm{d}}}{G_{\mathrm{d} 0}} C_{\mathrm{A}} \geqslant 1$

The inequality (4) represents the principal starting point for the construction of the sizing curve, based - for the moment — on a single climatic cycle. To demonstrate this procedure, let us consider the PV system configurations which satisfy the inequality (4). A particularly clear way to do this is to define a plane where a certain system configuration $\left(C_{\mathrm{S}}, C_{\mathrm{A}}\right)$ is represented by a point with the corresponding Cartesian coordinates (Fig. 1a). In this diagram, the systems that comply with the inequality (4) lie in the shaded area of the plane. It is interesting to note that the formalism that emerges resembles the methodology which has been used to discuss the sizing of hybrid PV-wind energy systems (Markvart, 1996).

It is now a simple matter to extend this method to describe real life situations. Fig. 2a shows the daily solar radiation in London during a part of the win-

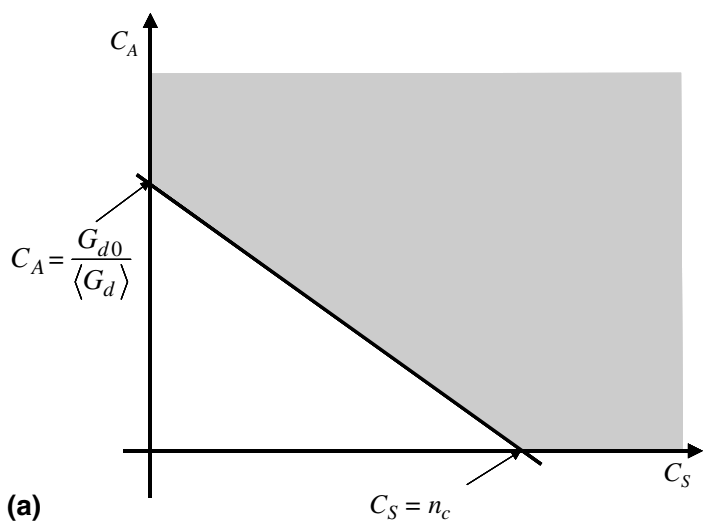

(a)

$$
C_{S}=n_{c}
$$

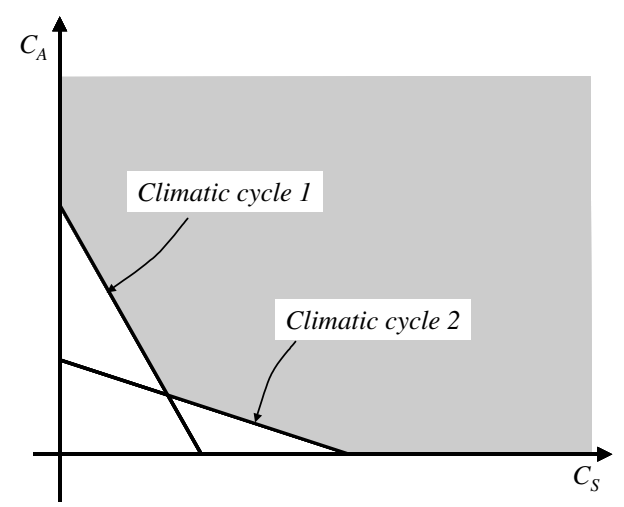

(b)

Fig. 1. A graphical method of solution for the inequality (4). System configurations that comply with the inequality (4) lie in the shaded area of the plane. System configurations that provide continuous power during a single climatic cycle (a) and during two climatic cycles (b).

ter 1989-1990 (Scharmer and Grief, 2000). It is seen that, for much of the time between 1st December and 7 th January, the daily radiation is below the long term average value for the location $\left(0.87 \mathrm{~kW} \mathrm{~h} / \mathrm{m}^{2}\right)$. A quick estimate of the energy balance in a system designed for the average radiation (Fig. 2b) indeed shows that there is a deficit in the energy supply for a considerable period of time, which has to be bridged over by the battery. Assuming that the battery is fully charged at the beginning of this cycle (as confirmed by detailed modelling), the battery size is determined by the energy deficit at the end of the climatic cycle (the last day of below-average radiation). We note, however, that it takes considerably longer before the battery is fully charged again. We can again use the inequality (4) to calculate the required battery size if $G_{d}$ is replaced by the average solar radiation during the climatic cycle. Thus, the inequality (4) provides a simple means to determine the system configuration 


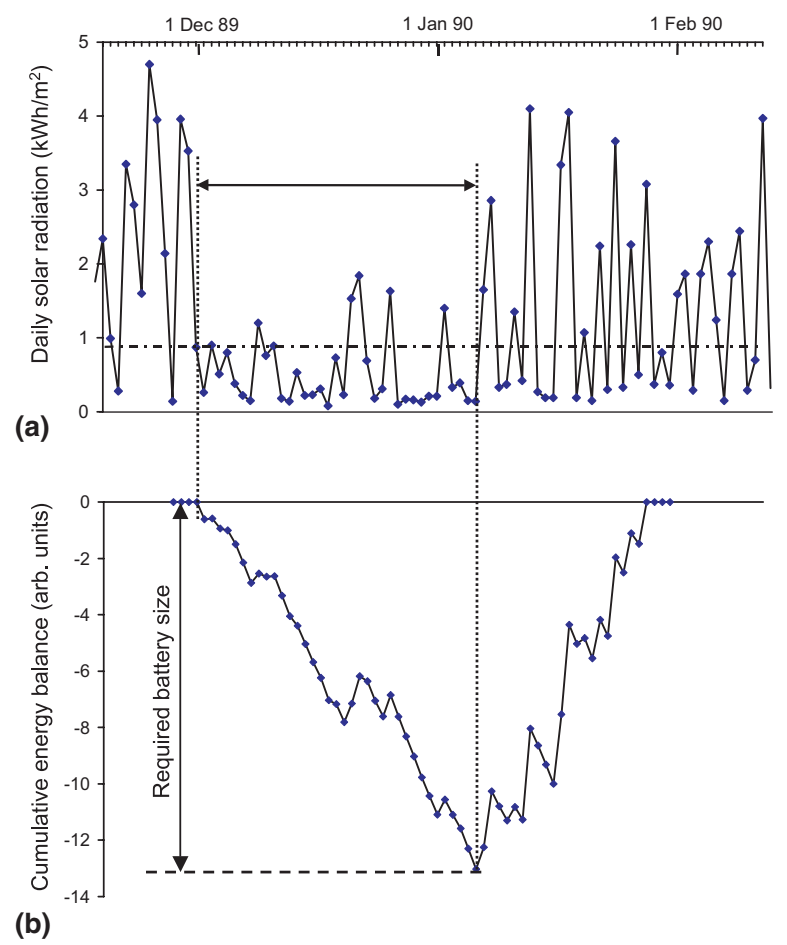

Fig. 2. (a) The daily solar radiation in London during the winter of 1989-1990, showing the dominant climatic cycle extending from 1st December 1989 to 7th January 1990. The average daily radiation $G_{\mathrm{d} 0}$ (shown by the dash-dot line) is the long mean value for December. (b) The cumulative energy balance (energy taken out of the battery) for a system design based on the average daily radiation in December.

which would be needed to provide a continuous solar electricity supply during the winter of 19891990.

To extend this formalism to embody information about solar radiation over a longer period of time, all we need to do is to consider the relevant climatic cycles during each year, and plot the resulting lines corresponding to several climatic cycles on the same graph (Fig. 1b). The same procedure but using actual solar radiation data results in a "sizing curve" is shown in Fig. 3. It is seen that only some years appear on the graph - the missing years are those with relatively high solar radiation and/or short climatic cycles. The area above the line describes system configurations which would have delivered uninterrupted power to the load during the period in question - in this case, during the years 1980-1990.

It is natural to speculate on the shape of the sizing curve if different time series of ten-year duration were used in the construction. A likely possibility that springs to mind is that the different

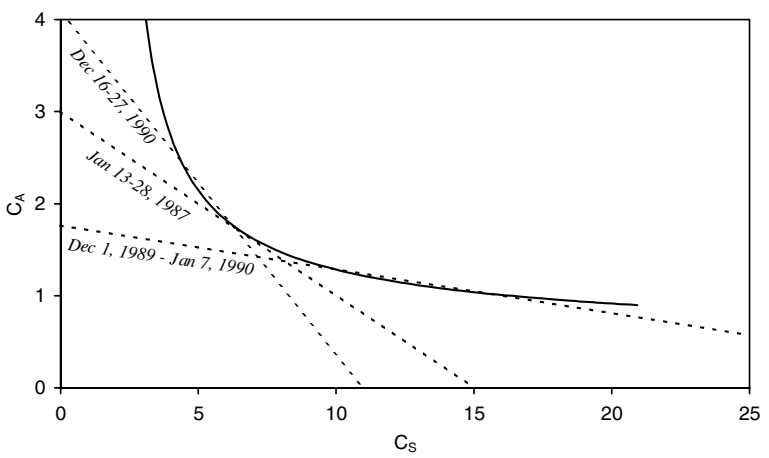

Fig. 3. The "sizing polygon" obtained by combining the three most prominent climatic cycles during the 1980-1990 decade. The full smooth line represents the dependence (5).

sizing lines would gradually define a sizing curve to which they are tangent. Such curve-shown in Fig. 3 by the smooth line-was recommended in Silvestre (2003) by using a simple two-parameter dependence

$C_{\mathrm{A}}=\frac{1}{a \ln C_{\mathrm{S}}-b}$

with $a=0.4514$ and $b=0.2601$. A more systematic approach might involve a dependence between the average solar radiation $G_{\mathrm{d}}$ and the duration $n_{\mathrm{c}}$ of the climatic cycle, for extreme time series of solar radiation. An preliminary indication of the effect of climate variability on PV system sizing is given by Fragaki and Markvart (2005).

It is clear that the procedure described above (in particular, Eq. (5) is applicable only to the location where the data have been obtained-in this instance, the South East of England. The results shown in Fig. 3 are in keeping with the usual practice of installing 15 days of storage with a PV array, obtained with the use of Eq. (1), and corresponding to the mean daily radiation $G_{\mathrm{d} 0}$ for December (see, for example, www.bpsolar.com).

\section{Conclusions}

This paper has discussed how a sizing curve for a PV system can be constructed based on the time series of observed solar radiation data over a certain period of time. By using a simple geometrical construction we have shown how the data can be used to determine system configurations that would be expected to deliver energy to load without interruption over the period of time in question.

It should be stressed that although the systems are designed for operation without shedding load, 
they cannot be assigned the LLP value of zero (if such reliability can ever be achieved!) as loss of supply may occur if the system operates over a longer duration than the design period of time.

In comparison with the methods based on loss of load probability (Egido and Lorenzo, 1992), this method takes a more pragmatic approach, and describes a technique for the construction of a sizing curve using a measured time series of solar radiation data, over a duration for which the data are available. Clearly, a higher reliability of supply may be achieved by considering longer time series of data. In this respect, the present method is similar to techniques used in other branches of engineering where reliability is considered as a function of recurrence intervals.

Although the resulting curve resembles the sizing curve determined by probabilistic arguments using synthetic solar radiation profiles it is difficult at this point to make a quantitative link between the length of the time series of data and the values of LLP. The LLP methods assign a probability value to project past solar radiation into the future; the present method extracts the essential information from the past data to determine the impact on the operation of the PV system. Since both types are based on solar radiation from the past they are equally good (or equally bad) in predicting the future behaviour (see also Fragaki and Markvart, 2005, where this point is made more explicitly). We hope to return to the link between solar radiation and PV system sizing in a separate publication.

\section{Acknowledgements}

This paper would not have seen the light of day were it not for the many enlightening discussions with Eduardo Lorenzo and Miguel Angel Egido many years ago. We are also grateful to John Page for his introduction to the European Solar Radiation Atlas, and his patient explanation of its many uses.

\section{References}

Barra, L., Catalanotti, S., Fontana, F., Lavorante, F., 1984. An analytical method to determine the optimal size of a photovoltaic plant. Solar Energy 33, 509.

Bartoli, B., Cuomo, V., Fontana, F., Serio, C., Silvestrini, V., 1984. The design of photovoltaic plants: an optimization procedure. Applied Energy 18, 37.

Bucciarelli, L.L., 1984. Estimating loss-of-power probabilities of stand-alone photovoltaic energy systems. Solar Energy 32, 205.

Bucciarelli, L.L., 1986. The effect of day-to-day correlation in solar radiation on the probability of loss-of-power in a standalone photovoltaic energy system. Solar Energy 36, 11.

Castañer, L., Bermejo, S., Markvart, T., Fragaki, A., 2003. Energy production by a PV array. In: Markvart, T., Castañer, L. (Eds.), Practical Handbook of Photovoltaics: Fundamentals and Applications. Elsevier, Oxford, p. 517.

Castillo, E., 1988. Extreme Value Theory in Engineering. Academic Press, London/San Diego.

Egido, M.A., Lorenzo, E., 1992. Review of sizing and a proposed new method. Solar Energy Materials and Solar Cells 26, 51.

Fragaki, A., 2005. Analysis of a new approach to sizing standalone PV systems. PhD Thesis, University of Southampton.

Fragaki, A., Markvart, T., 2005. Does global warming affect the design of PV systems? Progress in Photovoltaics: Research and Applications 13, 635.

Gordon, J.M., 1987. Optimal sizing of stand alone photovoltaic solar power systems. Solar Cells 20, 295.

Klein, S.A., Beckman, W.A., 1987. Loss-of-load probabilities for stand-alone photovoltaic systems. Solar Energy 39, 499.

Lorenzo, E., Araujo, G.L., Cuevas, A., Egido, M.A., Minano, J.C., Ziles, R., 1994. Solar Electricity: Engineering of Photovoltaic Systems, Progensa.

Markvart, T., 1996. Sizing of hybrid photovoltaic-wind energy systems. Solar Energy 57, 277.

Scharmer, K., Grief, J., 2000. European Solar Radiation Atlas, fourth ed., Les Presses de l' École des Mines, Paris.

Silvestre, S., 2003. Review of system design and sizing tools. In: Markvart, T., Castañer, L. (Eds.), Practical Handbook of Photovoltaics: Fundamentals and Applications. Elsevier, Oxford, p. 543. 